\title{
Circadian rhythms, sleep, and metabolism
}

\author{
Wenyu Huang,1,2 Kathryn Moynihan Ramsey,1,2 Biliana Marcheva,1,2 and Joseph Bass,1,2 \\ ${ }^{1}$ Department of Medicine, Feinberg School of Medicine, Northwestern University, Chicago, Illinois, USA \\ 2Department of Neurobiology and Physiology, Northwestern University, Evanston, Illinois, USA.
}

\begin{abstract}
The discovery of the genetic basis for circadian rhythms has expanded our knowledge of the temporal organization of behavior and physiology. The observations that the circadian gene network is present in most living organisms from eubacteria to humans, that most cells and tissues express autonomous clocks, and that disruption of clock genes results in metabolic dysregulation have revealed interactions between metabolism and circadian rhythms at neural, molecular, and cellular levels. A major challenge remains in understanding the interplay between brain and peripheral clocks and in determining how these interactions promote energy homeostasis across the sleep-wake cycle. In this Review, we evaluate how investigation of molecular timing may create new opportunities to understand and develop therapies for obesity and diabetes.
\end{abstract}

\section{Introduction: circadian clocks and metabolism from molecular systems to physiology}

Studies in plants dating from the 18th century originally established that 24-hour periodic phenomena arise from biological oscillators that internally track the rotation of the Earth. These so-called "circadian clocks" (the term is derived from "circa diem," or "about a day") are entrained by light and synchronize energy-harvesting and utilization processes with the rising and setting of the sun (ref. 1 and Figure 1A). At the molecular level, circadian rhythms are encoded by an autoregulatory loop composed of a set of transcription activators (CLOCK:NPAS2/ BMAL1 [MOP3]) that induce expression of repressors (PER1-3/ CRY1-2) that feedback to inhibit the forward limb. Discovery of this mechanism stemmed from deliberate mutagenesis experiments in flies (2), and forward genetics in mice ultimately led to the discovery of the first mammalian Clock gene (3-5). Subsequent work has revealed that the mammalian molecular clock is not only expressed within the master suprachiasmatic nucleus (SCN) pacemaker neurons, but also within nearly all cells $(6,7)$. In addition to this core loop, CLOCK/BMAL1 also induce expression of the orphan nuclear hormone receptors (NHRs) ROR $\alpha$ and REV-ERB $\alpha$, which activate and repress Bmal1 transcription, respectively. Posttranslational regulation of clock transcription factors by casein kinase I $\varepsilon$ and $\mathrm{I} \delta(\mathrm{CKI} \varepsilon / \delta), \beta-\operatorname{TrCP}$, FBXL3, and ARF-BP1/PAM, in addition to cAMP signaling (8), further modulates circadian oscillations (1). The purpose of this Review is to provide an overview of the genetic and neurobiological evidence linking circadian and energetic systems and to highlight gaps in our understanding of the molecular pathways that couple these processes.

\section{Neural networks linking circadian and energetic centers}

In vertebrates, circadian systems are organized hierarchically with master pacemaker neurons within the hypothalamic SCN presiding over a distributed network of extra-SCN and peripheral clocks (9). The environmental light-dark cycle provides the principal entraining signal to the SCN, which in turn produces synchronized rhythms of behavior and physiology through alignment of circadian gene oscillation within both extra-SCN neu-

Conflict of interest: Joseph Bass is a member of the scientific advisory board of ReSet Therapeutics Inc. and has received support from Amylin Pharmaceuticals.

Citation for this article: J Clin Invest. 2011;121(6):2133-2141. doi:10.1172/JCI46043. rons and peripheral tissues (Figure 1B). Wiring of the neural circuit generating circadian rhythms involves projections of the SCN to a wide range of cell bodies within both the hypothalamus (including the arcuate nucleus [ARC], paraventricular nucleus [PVN], lateral hypothalamic area [LHA], and dorsomedial hypothalamus [DMH]; refs. 10-16) and the brainstem (including the ventral tegmental area [VTA] and dorsomedial nucleus of vagus $[\mathrm{DMV}]$ via relay at the medial preoptic area [MPO] and PVN, respectively refs. 17,18 ) (Figure 2 ). Within the ARC, neurons expressing orexigenic neuropeptide Y/Agouti-related protein (NPY/AgRP) and anorexigenic pro-opiomelanocortin/cocaineand amphetamine-regulated transcript (POMC/CART) peptides form reciprocal connections with cells located within the SCN $(10,11)$. In addition, NPY/AgRP- and POMC/CART-expressing neurons and SCN neurons project to cell bodies within the PVN and LHA that modulate autonomic (18) and sleep-wakefulness $(19,20)$ behavior, respectively. While peptidergic signaling within NPY/AgRP and POMC/CART neurons has been shown to influence long-term body weight constancy (see refs. 21-24 for more complete reviews), less is known concerning circadian modulation of signaling within these cells. For instance, clock genes are expressed cell-autonomously within cell bodies that receive projections from the SCN; thus it is possible that oscillation of key transcripts important in the response to anorexigenic and orexigenic hormones may be subject to circadian control. Interestingly, feeding an isocaloric high-fat diet at the incorrect circadian time has been shown to result in increased weight gain in mice (25), suggesting that circadian alignment of feeding and activity is critical in the homeostatic control of body weight. It is also intriguing that projections originating in the SCN directly synapse at the orexin-producing (ORX-producing, also referred to as hypocretinproducing) neurons within the LHA $(14,26,27)$. ORX neurons in the LHA are important in peripheral glucose metabolism (28) and also participate in long-term weight homeostasis, since ORX receptor knockout mice are susceptible to diet-induced obesity (29). Finally, since dopaminergic signaling in the VTA neurons is implicated in reward systems associated with feeding $(30,31)$, an intriguing question is whether hedonic drive might similarly display circadian variation (32). Further studies are needed to define the repertoire of connections between SCN and energetic neurons, to determine the impact of feeding rhythms on function and entrainment of extra-SCN clocks, and to elucidate how clocks participate in melanocortinergic and dopaminergic signaling. 


\section{A Cyclic energy harvesting}

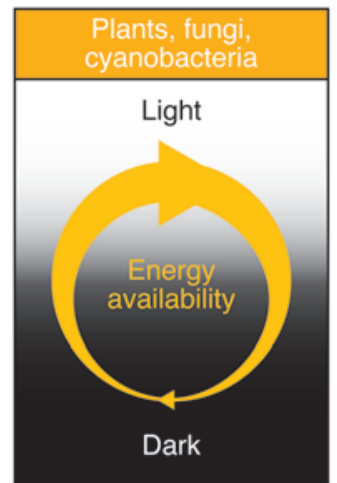

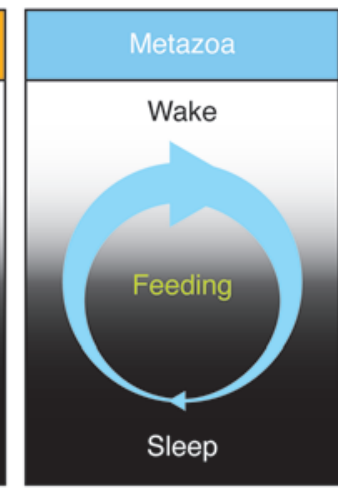

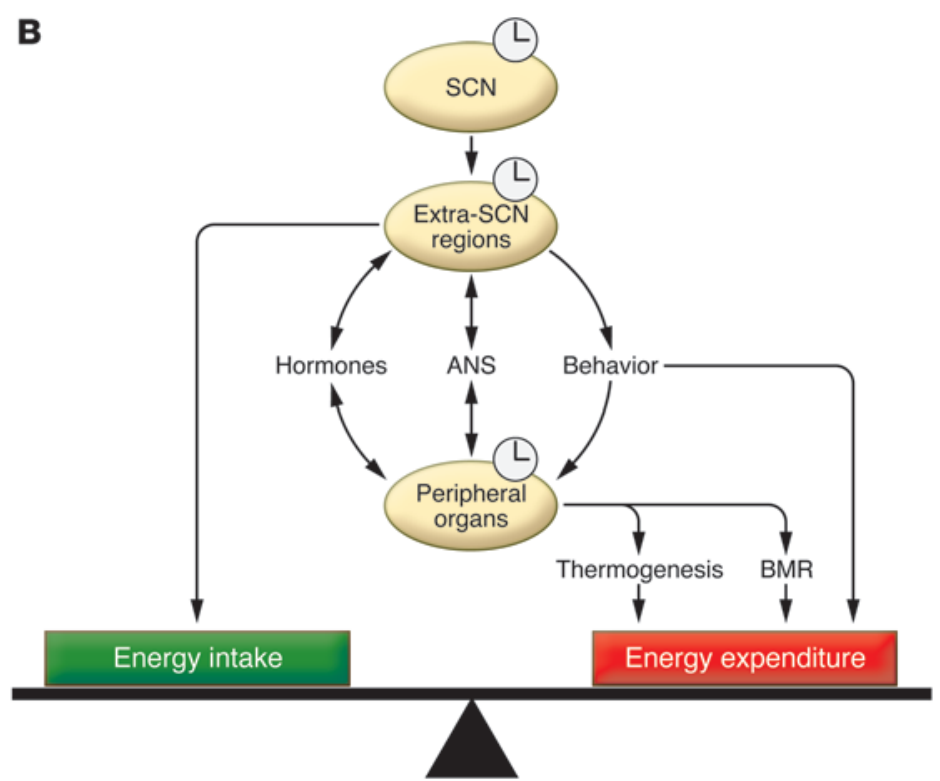

Figure 1

Circadian control of energy metabolism. (A) In plants, cyanobacteria, and fungi, energy is available during the light period within the light/dark cycle, while in metazoa, alternating periods of sleep and wakefulness, closely associated with the light/dark cycle, impart cyclicity on feeding behavior and fuel utilization. (B) The master clock in the SCN sends signals to the extra-SCN regions, which in turn entrain peripheral tissues via hormonal, autonomic nervous system (ANS), and behavioral pathways in order to regulate peripheral clock control of fuel utilization and energy harvesting. Extra-SCN regions also regulate energy homeostasis by controlling cyclic energy intake and locomotor activity. Through regulation of food intake, physical activity, and metabolic processes, both brain and peripheral clocks contribute to long-term weight stability by maintaining a precise balance between energy intake and energy expenditure. A positive energy balance deposits stored energy mostly into adipose tissue, leading to obesity, while a negative energy balance results in leanness. BMR, basal metabolic rate.

\section{Interaction of circadian and metabolic systems at the cell and molecular levels}

Integration of circadian systems with the light/dark environment involves a widely distributed network of local tissue clocks within both the brain and periphery (33-36) (Figure 3). Interestingly, a variety of factors including food availability, glucocorticoid level, and temperature, so called "zeitgebers" ("time givers"), are able to reset the phase of peripheral clocks. In contrast, the SCN is entrained only to light but not to feeding; thus shifts in feeding time uncouple the phase of peripheral tissue clocks from that of the SCN (37-40).

Feedback loops between nutrient sensors and molecular clocks in peripheral tissues (NAD ${ }^{+}, N A M P T$, SIRT1, AMPK). Recent studies have demonstrated that various nutrient sensors are able to relay information regarding the cellular nutrient status to the circadian clock. For example, early studies indicated that the reduced forms of the redox cofactor NADH increase CLOCK/BMAL1 and NPAS2/BMAL1 heterodimer activity, whereas the oxidized forms $\left(\mathrm{NAD}^{+}\right)$inhibit their activity (41). The $\mathrm{NAD}^{+}$-dependent deacetylase sirtuin 1 (SIRT1), which is induced by acute nutrient withdrawal (42) or calorie restriction (43), also directly binds to the CLOCK/BMAL1 complex to regulate expression of clock genes (44-47). AMP kinase (AMPK) serves as another highly conserved cellular nutrient sensor that, when activated by exercise, fasting, or hypoxia (48), leads to phosphorylation and degradation of CRY1 (49), as well as activation of NAMPT, the rate-limiting enzyme in $\mathrm{NAD}^{+}$salvage biosynthesis (50). Interestingly, the connection between nutrient sensors and molecular clocks is bidirectional. Levels of NAMPT, as well as $\mathrm{NAD}^{+}$itself, exhibit circadian oscillations, and Nampt gene transcription is directly regulated by CLOCK $(46,47)$. As such, SIRT1 activity is indirectly regulated by the clock network. Together, these data demonstrate on a molecular level that there is a complex crosstalk between highly conserved nutrient sensors and the molecular clock networks, at least at the level of peripheral tissues (see below for discussion regarding potential roles of CNS nutrient sensors in circadian control of metabolism).

Metabolic transcription factor and transcriptional co-activator feedback on the core clock in peripheral tissues (REV-ERB, ROR, PPAR, $D B P, P G C 1 \alpha)$. NHRs are ligand-activated transcription factors, a majority of which, including REV-ERB $\alpha$, RORs and PPARs, demonstrate circadian expression in peripheral tissues (51). REV-ERB $\alpha$ regulates hepatic gluconeogenesis, adipocyte differentiation, and lipid metabolism, and also represses Bmal1 transcription (52, 53). ROR $\alpha$ competes with REV-ERB $\alpha$ in binding to the Bmal1 promoter and induces Bmal1 expression (54) in addition to regulating lipid metabolism (55). PPAR $\alpha$, when activated by endogenous fatty acids (FAs), stimulates FA oxidation, regulates genes controlling lipid homeostasis, and prevents atherosclerosis (56). In addition, PPAR $\alpha$ positively regulates Bmal1 expression, while BMAL1 likewise activates PPAR $\alpha$, generating a positive feedback loop (57). Another subtype, PPAR $\gamma$, plays an important role in adipocyte differentiation and triglyceride synthesis (58). PPAR $\gamma$ induces Bmal1 expression in the blood vessels, and vasculature-specific PPAR $\gamma$ knockout leads to marked reduction in the circadian variation in blood pressure and heart rate (59).

In addition to NHRs, $D b p$, a known clock target gene, regulates expression of key metabolic genes involved in gluconeogenesis and lipogenesis (60). Because DBP levels change 100-fold in response 


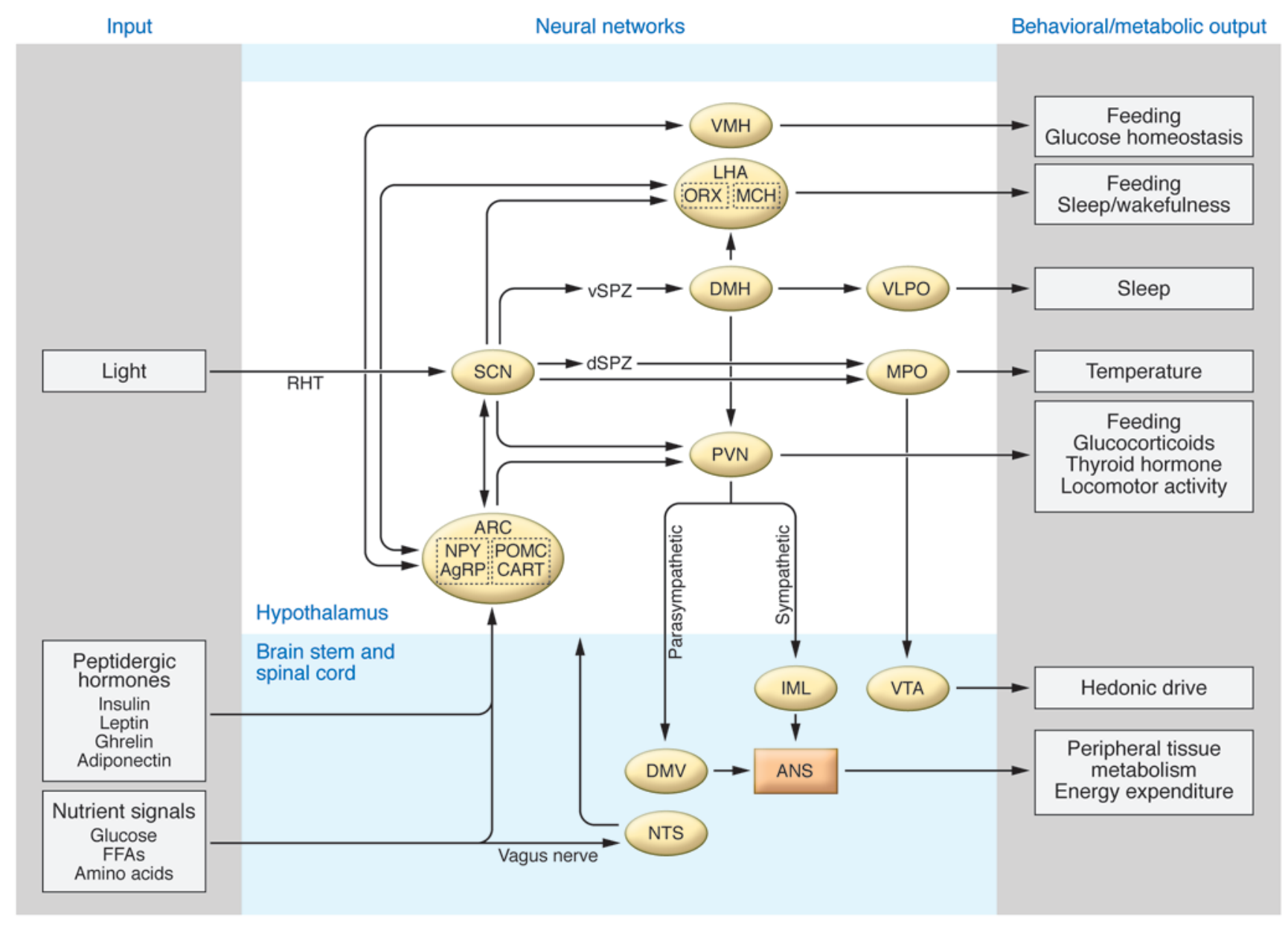

Figure 2

Map of neural circuits linking SCN and extra-SCN regions important in circadian and energetic control. CNS centers receive dual input of light and metabolic signals. Light reaches the SCN via the RHT, which in turn sends neural projections to various extra-SCN regions in the hypothalamus and brainstem that are critical for energy homeostasis and sleep, including the ARC, PVN, and ventrolateral preoptic nucleus (VLPO). The hypothalamus also receives metabolic inputs, including peptidergic hormones and nutrient metabolites, which modulate the CNS signaling. Thus signals from the exogenous environment (i.e., light) and endogenous metabolism (i.e., metabolic cues) are integrated in the CNS, the output of which in turn imparts rhythmicity on sleep and a variety of metabolic outputs, such as thermogenesis, feeding behavior, hormone secretion, and locomotor activity. IML, intermediolateral nucleus; NTS, nucleus tractus solitarius. dSPZ, dorsal subparaventricular zone; RHT, retinohypothalamic tract; vSPZ, ventral subparaventricular zone; $\mathrm{MCH}$, melanocyte concentrating hormone.

to CLOCK/BMAL1 activation, it is conceivable that DBP generates circadian oscillation in metabolic processes such as gluconeogenesis. Additionally, the transcriptional co-activator PPAR $\gamma$ co-activator $1 \alpha$ (PGC-1 $\alpha)$ (61) also displays circadian oscillations and regulates Bmal1 and Rev-erba expression. Knockout of Pgc1a leads to abnormal diurnal rhythms of activity, body temperature, and metabolic rate, in addition to aberrant expression of clock and metabolic genes (62). Interestingly, SIRT1 suppresses PPAR $\gamma$ (63) but activates PGC-1 $\alpha$ (64), and thus affects the clock network through multiple mechanisms.

Possible molecular integrators of circadian and metabolic systems in the CNS. Molecular analyses of interplay between circadian and metabolic pathways have primarily emerged from studies in liver and other peripheral tissues, yet it remains uncertain whether similar mechanisms might also couple these processes within the brain. One question is whether specific metabolites that vary according to time of day and nutrient state (fasting vs. feeding), may also affect circadian function of energy-sensing neurons. For instance, 24-hour oscillation in levels of glucose and FAs may in turn influence expression of circadian genes and rhythmic transcriptional outputs within hypothalamic neurons involved in glucose homeostasis (65), food intake (66), and energy expenditure (67-70). Perturbation of metabolic homeostasis with a high-fat diet is sufficient to alter both period length and amplitude of locomotor activity (71). These observations indicate that changes in FA metabolism per se may either alter clock gene function within SCN pacemaker neurons and/or interrupt communication between SCN and extra-SCN neurons.

Additional nutrient factors that may participate in circadian oscillations of SCN and extra-SCN neurons include AMPK, SIRT1, and the mammalian target of rapamycin (mTOR). Hypothalamic AMPK is regulated by nutrient state and hormones such as leptin and insulin (72), and manipulation of its expression alters food intake and body weight (73); however the impact of AMPK on CNS control of locomotor behavior and physiologi- 


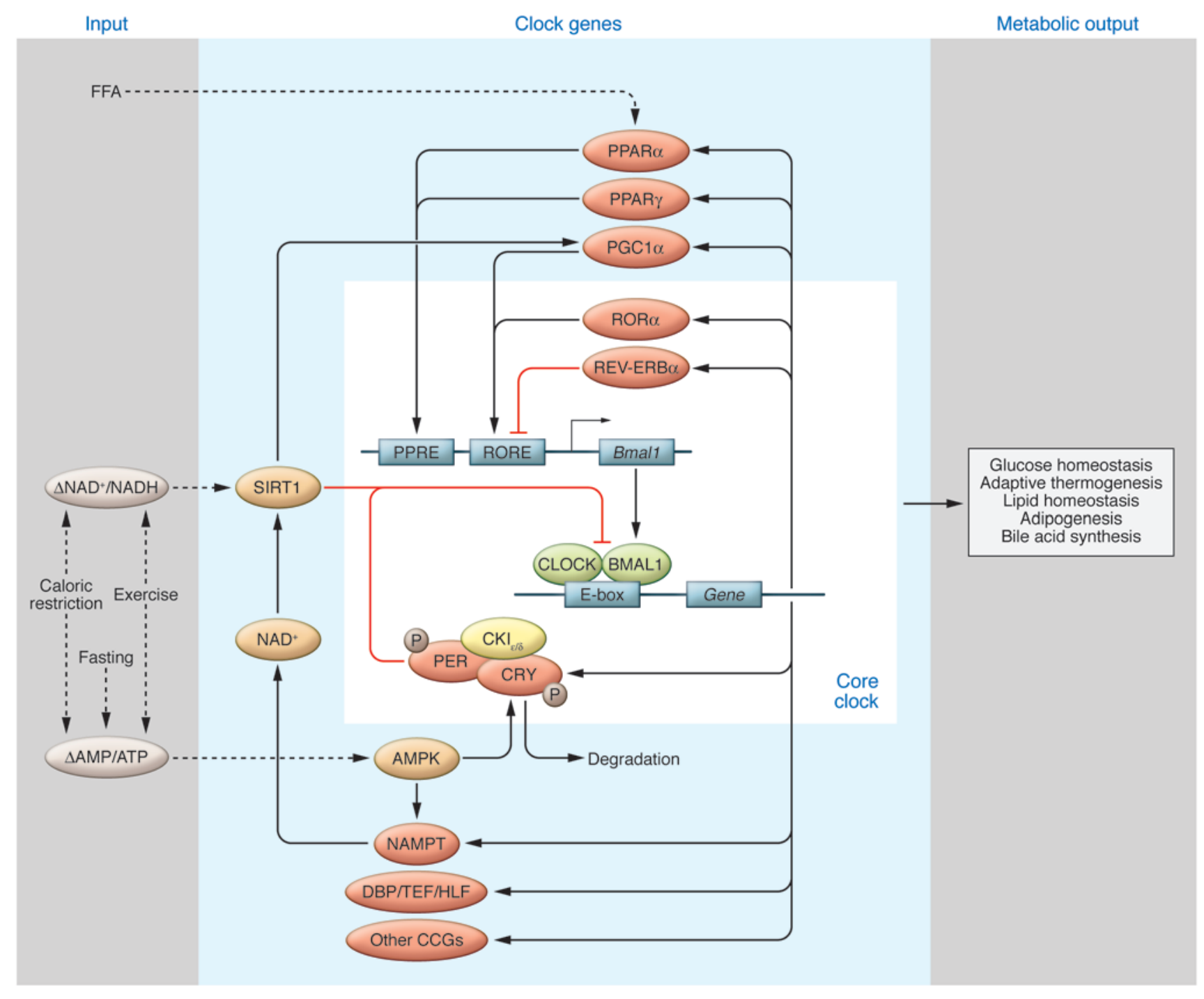

\section{Figure 3}

Interactions between the molecular clock and downstream metabolic genes. The core molecular clock consists of several transcription/translation feedback loops, including posttranscriptional regulation (yellow), that oscillate with an approximately 24-hour periodicity. CLOCK and BMAL1 heterodimerize to drive rhythmic expression of downstream target genes (shown in red), which in turn regulate diverse metabolic processes, including glucose metabolism, lipid homeostasis, and thermogenesis. Many of these clock target genes in turn reciprocally regulate the clock in response to changes in nutrient status (shown in blue) via cellular nutrient sensors (shown in orange), generating a complex network of interlocking feedback loops that fine-tune the clock and coordinate metabolic processes with the daily cycles of sleep/wakefulness and fasting/feeding. Dashed lines represent metabolic inputs; solid lines depict interactions among core clock genes, clock-controlled genes, and nutrient sensors.

cal rhythms is still not known. NPY and POMC neuron-specific knockout of the $\alpha 2$ subunit of AMPK results in lean and obese phenotypes, respectively, so it will be interesting to learn whether AMPK also participates in synchronizing activity and feeding through actions within these cell groups (74). SIRT1 may also represent an additional mechanism coupling nutrient flux with rhythmic activity of hypothalamic neurons, as the nutrient-sensing deacetylase is present within both ARC and DMH/LHA neurons $(75,76)$. A final potential mediator involved in both energy sensing and circadian function in the CNS is mTOR, a regulator of protein synthesis present in ARC neurons that has been shown to modulate food intake (77). MTOR is also expressed within pacemaker neurons of the SCN, where its expression is activated by light (78). Moreover, activation of mTOR causes phase resetting of SCN explants (79), while inhibition of mTOR alters light induction of the Period gene within the SCN of the intact animal (78). Thus amino acid metabolism may participate in both entrainment of the master clock and in the temporal organization of feeding.

Nutrient signaling, whether through glucose, FAs, AMPK, SIRT1, or mTOR, may function to entrain or "gate" CNS and peripheral clocks, leading to tissue-specific differences in phase and amplitude of gene expression rhythms. Indeed, different zeitgebers may exert differing effects on circadian entrainment in the brain and peripheral tissues, leading to distinct effects on phase and amplitude of gene oscillation within these locales. It is also possible that 
entrainment of peripheral clocks by specific hormones may be phase dependent (80); that is, local tissue clocks may only respond to phase-resetting hormones and/or metabolites when these are present within a narrow window of time during the 24 hour light/ dark cycle. For instance, glucocorticoid secretion from adrenal glands in response to adrenocorticotropin is dependent on time of day (81). In addition to metabolite and hormonal input into the clock, recent studies by Buhr et al. suggest that temperature and heat shock signaling pathways play a central role in entrainment of peripheral tissues (39). Importantly, since alteration in temperature and/or the heat shock pathway is closely associated with other synchronizing signals including feeding and glucocorticoid secretion, it may serve as a unifying signal in entraining peripheral clocks. A major objective in future research will thus be to delineate the role of nutrient signaling in entrainment of CNS and peripheral clocks, and to determine how these signals interact with synchronizing signals such as feeding and neuroendocrine hormones (e.g., glucocorticoids) to maintain phase alignment of behavioral and metabolic rhythms within the whole organism at the levels of both physiology and pathophysiology.

\section{Role of the circadian system in energy balance, metabolism, and sleep}

Evidence for circadian integration of energetics, metabolism, and sleep in bumans. In humans, many aspects of metabolism display circadian cycles, including 24-hour variation of glucose, insulin, and leptin levels $(82,83)$. Genome-wide association studies have also suggested connections between clock gene variation and fasting glucose levels $(84,85)$, obesity, and metabolic syndrome (86), raising interest in understanding the impact of circadian systems on human disease. One common clinical condition suggestive of interactions between circadian rhythms and metabolism in humans is that of shift work. Numerous reports have indicated that shift workers have a higher incidence of diabetes, obesity, and cardiovascular events $(1,87)$, although the mechanism underlying this association is uncertain. Scheer et al. recently tested the impact of forced circadian misalignment (a simulation of shift work) on neuroendocrine control of glucose metabolism and energetics (88). In participants subjected to circadian misalignment, the investigators observed hypoleptinemia, insulin resistance, inverted cortisol rhythms, and increased blood pressure (88). It is also interesting to note that patients with diabetes exhibit dampened amplitude of rhythms of glucose tolerance and insulin secretion (89); thus the relationship between circadian disruption and metabolic pathologies appears to be bidirectional in humans, suggesting that circadian disruption may lead to a vicious cycle and contribute to augmentation and progression of metabolic disease.

Direct genetic evidence in humans has linked the molecular clock with sleep $(90,91)$ through the positional cloning of mutations causing familial advanced sleep phase syndrome, which is characterized by early sleep onset and awakening (92). In the general population, observational studies have also found that short sleep, sleep deprivation, and poor sleep quality are associated with diabetes, metabolic syndrome $(82,93)$, hypoleptinemia, increased appetite, and obesity $(94,95)$. A recent study showed that sleep duration correlates with the magnitude of weight loss as fat in response to caloric restriction; short sleepers appear to have more difficulty losing fat compared to long sleepers despite similar amount of weight loss (96). Narcolepsy, a sleep disorder in which patients present with extreme daytime sleepiness due to loss of hypocretin-producing neurons $(97,98)$, has been associated with elevated BMI (99) and increased incidence of obesity $(100,101)$. Night eating syndrome (NES) is another instance in which disrupted rhythmic patterns of sleep and eating correlate with altered metabolism (102) and obesity (103). Patients with NES consume significantly more of their daily energy intake at night, although their total daily food intake is similar to that of control subjects $(104,105)$. They also have abnormal rhythms of metabolic hormones, including decreased nocturnal rise in leptin, a phase shift in insulin, cortisol, and ghrelin, and inverted 24-hour rhythms of blood glucose (104, 106). Interestingly, the nocturnal pattern of eating observed in NES patients is reminiscent of feeding alterations in the Clock mutant mouse. These animals exhibit increased feeding during the normal sleep period together with increased susceptibility to diet-induced obesity (107). A major goal is to determine whether the adverse metabolic consequences of sleep loss (and accompanying feeding alterations) are due to the disrupted circadian rhythms per se, to the altered sleep, or to some combination of the two. Nonetheless, the above observations in humans demonstrate that synchronization of feeding/fasting and active/rest periods with the environmental light/dark cycle influences body weight constancy.

Integration of circadian rhythms and energy homeostasis in animal models. Studies in rodents have also attempted to experimentally simulate shift work in order to further probe mechanisms linking circadian disruption with metabolic disorders. When rats were exposed to a daily eight-hour activity schedule during their normal resting phase, they succumbed to diminished rhythms of glucose and locomotor activity, as well as obesity, which correlated with increased food intake during their resting phase (108). However, shifting food intake back to the active phase restored their metabolic rhythms and prevented obesity in these same animals (108), suggesting that the normal alignment of feeding and activity with the environment light cycle is critical for the maintenance of energy homeostasis. Furthermore, feeding wild-type mice ad libitum exclusively during the daytime resulted in greater weight gain than in animals fed exclusively at night (25). Similarly, when genetically obese mice with disrupted diurnal feeding patterns were fed exclusively at night, their obesity and metabolic disorders improved (109).

Genetic animal models of clock gene disruption have provided an additional approach to specifically dissect the effects of molecular clock genes on energy homeostasis. For example, Clock mutant mice are obese and hyperphagic (107), although this phenotype may be modified by genetic background (110). Moreover, introgression of the Clock mutation into the $o b / o b$ strain exacerbates obesity (111). In addition to causing metabolic disorders, both

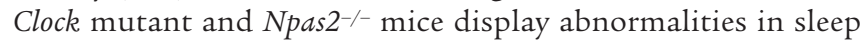
architecture, so it will be critical to determine the cause-and-effect relationship between alterations in sleep and feeding in these models $(112,113)$. In addition, Npas $2^{-/-}$mice display increased activity during the "siesta" that wild-type mice normally take during the mid-active period (112). In contrast, Clock mutants have increased locomotor activity and food intake during the period when wild-type mice are at rest and fasting (107), suggesting nonredundant functions between the Clock and Npas 2 paralogs with regard to energy balance. Global Bmal1 knockout mice are arrhythmic and display increased adiposity at early ages (114); however, these animals also develop arthropathy and myopathy, resulting in decreased activity and weight (115). Interestingly, brain rescue of Bmal1-knockout mice only restores behavioral circadian rhythms, whereas muscle rescue restores activity levels and body weight, but 
not behavioral rhythms $(116,117)$, suggesting that BMAL1 function within the brain controls period length and activity rhythms, whereas its expression in muscle affects mitochondrial function and exercise tolerance (117). Curiously, mice with mutations in the Period genes display increased adiposity (118). Future application of neuron-specific targeting approaches will be important to identify selective effects of circadian gene disruption on the interrelation between sleep and energy homeostasis.

Integration of circadian rhythms and glucose homeostasis in animal models. Glucose homeostasis is under circadian control at the level of both peripheral tissues and the SCN. Ad libitum glucose levels peak and glucose tolerance is enhanced at the beginning of the active phase compared with the rest phase $(119,120)$. The morning peak of glucose is postulated to result from increased hepatic gluconeogenesis (120) as well as low insulin secretion (121), whereas the improved glucose tolerance (early in the active period) is likely due to elevated glucose uptake at skeletal muscle and adipose tissues $(119,122)$. Genetic disruption of components of the clock network has identified a role of core clock genes in glucose homeostasis. For example, multi-tissue Clock mutant mice develop age-dependent hyperglycemia and hypoinsulinemia $(107,123)$, in part due to impaired insulin secretion and defects in proliferation of pancreatic islets. Cry-knockout animals have increased hepatic gluconeogenesis, in part due to upregulation of cAMP signaling (124). A major advance toward understanding the tissue-specific roles of peripheral clocks in glucose homeostasis came with the development of conditional gene-targeting approaches. While deletion of Bmal1 within liver causes hypoglycemia (114), deletion of Bmal1 within pancreas causes hyperglycemia and hypoinsulinemia (123); thus at the physiological level, the actions of clock activator genes in the liver oppose those in the pancreas. Interestingly, both Clock mutant and Bmal1knockout mice are hypersensitive to insulin, although the mechanism for this remains to be defined (115). While the above genetic models demonstrate a role for peripheral clocks in regulation of glucose metabolism, the SCN also plays a critical role in controlling the circadian variation of glucose and glucose tolerance (119), in part through rhythmic modulation of autonomic nervous system efferents (125). An important remaining question is whether peripheral clocks are necessary for the proper alignment of behavioral states (sleep/wakefulness, fasting/feeding) with peripheral metabolism in liver, muscle, adipose tissue, and pancreas.

Integration of circadian rhythms and lipid homeostasis in animal models. In addition to glucoregulatory pathways, the circadian system also regulates lipid homeostasis and adipose tissue metabolism. Both intestinal lipid transport and de novo lipid synthesis exhibit circadian variation (126), as do levels of adipose tissue hormones such as adiponectin (127) and leptin (128). Hypertriglyceridemia is evident in Clock mutant mice (107), likely due to both intestinal overabsorption (129) and hepatic overproduction (130). One node of coupling circadian and lipogenic pathways involves REV-ERB $\alpha$ (52). REV-ERB $\alpha$ controls SREBP signaling and bile acid homeostasis, both of which are essential for lipid metabolism (131). Furthermore, BMAL1 is necessary for adipogenesis, as embryonic fibroblasts from Bmal1 knockout mice fail to differentiate into adipocytes (132). Clock genes may also indirectly regulate adipogenesis via PPARs. Finally, the SCN has also been shown to be critical for regulation of the diurnality of leptin release $(133,134)$. Further studies are necessary to assess the potential impact of clock genes on additional functions of the adipocytes, including thermogenesis and lipokine secretion.
Reciprocal effects of nutrient signaling on circadian rhythms and sleep. The relationship between circadian rhythms and metabolism is bidirectional; as noted above, a high-fat diet lengthens the intrinsic period length of locomotor activity, alters the circadian rhythms of feeding, reduces the amplitude, and shifts the phase of metabolic gene expression cycles in liver, fat, and hypothalamus (71). Similarly, genetically obese $o b / o b$ and $d b / d b$ mice $(135,136)$ have disrupted circadian rhythms of activity and sleep/wake patterns. In addition, glucocorticoid, a metabolic hormone involved in numerous biological processes such as gluconeogenesis, also entrains peripheral clocks (40) and increases expression of multiple clock genes, including Per1 and Per2 (137).

Restricted feeding studies have further suggested that nutrient conditions influence rhythmic locomotor activity and peripheral clock gene entrainment, although the role of clock genes per se in activity rhythms remains controversial. Whereas the period of locomotor activity programmed by the SCN remains constant even when food is restricted each day to the light period, foodrestricted animals display an anticipatory increase in locomotor activity (138), temperature, and glucocorticoid secretion that persists even when the food is removed (139). Moreover, food availability can entrain rhythms in peripheral tissues such as liver and kidney, but not in the $\operatorname{SCN}(37,38)$, suggesting the existence of a food-entrainable oscillator (FEO) (140). The anatomic location of the FEO has been a topic of intensive investigation, although efforts to understand the mechanism for this process have produced inconclusive results. While several studies have suggested that the DMH is necessary and sufficient to induce food-entrainable circadian rhythms $(141,142)$, other studies have questioned the role of the DMH as the FEO (143).

An important remaining question concerns the identity of the synchronizing signals that adjust circadian oscillations according to feeding time. Potential signals involved in resetting peripheral clocks are numerous, including glucose, lipids, sterols, peptidergic molecules, and catecholamines. Elucidating mechanisms involved in circadian entrainment of feeding may lead to improved interventions for clinical pathologies associated with shift work and jet lag.

\section{Concluding remarks}

Advances in genetic studies of circadian rhythms have led to the recognition that the circadian system is tightly coupled with processes controlling both sleep and metabolism. These dynamic interactions ensure that energy metabolism is coordinated in a proper temporal pattern and that circadian control is also subject to modulation by the energy status of the organism. Disruption of either the circadian clock or metabolism can lead to derangement of the other, thus predisposing to metabolic disorders such as obesity and type 2 diabetes. Future research will continue to focus on expanding our understanding of how brain and peripheral clocks coordinately regulate metabolic processes at both the cell-autonomous and non-autonomous level, how nutrient flux translates information regarding environmental milieu to the clock, and the impact of circadian rhythms in human health and disease.

\section{Acknowledgments}

We thank Ravi Allada, Shin-ichiro Imai, Joseph S. Takahashi, Fred W. Turek, and members of the Bass lab for helpful comments and discussions. K.M. Ramsey received support from NIDDK grant T32 DK007169. J. Bass is supported by NIH grants P01 AG011412 
and R01HL097817-01, the American Diabetes Association, Chicago Biomedical Consortium Searle Funds, the Juvenile Diabetes Research Foundation, and the University of Chicago Diabetes Research and Training Center (grant P60 DK020595). Joseph Bass is a member of the scientific advisory board of ReSet Therapeutics Inc. and has received support from Amylin Pharmaceuticals. The Obesity Review Series is supported in part by unrestricted educa- tional grants from Merck \& Co. and the Life Sciences Institute of the University of Michigan.

Address correspondence to: Joseph Bass, Northwestern University, 303 East Superior St., Lurie 7-107, Chicago, Illinois 60611, USA. Phone: 312.503.2258; Fax: 312.503.5453; E-mail: j-bass@ northwestern.edu.
1. Bass J, Takahashi JS. Circadian integration of metabolism and energetics. Science. 2010; 330(6009):1349-1354.

2. Konopka RJ, Benzer S. Clock mutants of Drosophila melanogaster. Proc Natl Acad Sci U S A. 1971; 68(9):2112-2116

3. King DP, et al. Positional cloning of the mouse circadian clock gene. Cell. 1997;89(4):641-653.

4. Vitaterna MH, et al. Mutagenesis and mapping of a mouse gene, Clock, essential for circadian behavior Science. 1994;264(5159):719-725.

5. Antoch MP, et al. Functional identification of the mouse circadian Clock gene by transgenic BAC rescue. Cell. 1997;89(4):655-667.

6. Panda S, et al. Coordinated transcription of key pathways in the mouse by the circadian clock. Cell. 2002;109(3):307-320.

7. Reddy $\mathrm{AB}$, et al. Circadian orchestration of the hepatic proteome. Curr Biol. 2006;16(11):1107-1115.

8. O'Neill JS, Maywood ES, Chesham JE, Takahashi JS, Hastings MH. cAMP-dependent signaling as a core component of the mammalian circadian pacemaker. Science. 2008;320(5878):949-953.

9. Weaver DR. The suprachiasmatic nucleus: a 25-year retrospective. J Biol Rhythms. 1998;13(2):100-112.

10. Saeb-Parsy K, Lombardelli S, Khan FZ, McDowall $\mathrm{K}$, Au-Yong IT, Dyball RE. Neural connections of hypothalamic neuroendocrine nuclei in the rat. J Neuroendocrinol. 2000;12(7):635-648.

11. Yi CX, van der Vliet J, Dai J, Yin G, Ru L, Buijs RM. Ventromedial arcuate nucleus communicates peripheral metabolic information to the suprachiasmatic nucleus. Endocrinology. 2006;147(1):283-294.

12. Berk ML, Finkelstein JA. An autoradiographic determination of the efferent projections of the suprachiasmatic nucleus of the hypothalamus. Brain Res. 1981:226(1-2):1-13.

13. Vrang N, Mikkelsen JD, Larsen PJ. Direct link from the suprachiasmatic nucleus to hypothalamic neurons projecting to the spinal cord: a combined tracing study using cholera toxin subunit B and Phaseolus vulgaris-leucoagglutinin. Brain Res Bull. 1997;44(6):671-680.

14. Abrahamson EE, Leak RK, Moore RY. The suprachiasmatic nucleus projects to posterior hypothalamic arousal systems. Neuroreport. 2001;12(2):435-440.

15. Sanchez-Lasheras C, Konner AC, Bruning JC. Integrative neurobiology of energy homeostasis-neurocircuits, signals and mediators. Front Neuroendocrinol. 2010;31(1):4-15.

16. Saper CB, Scammell TE, Lu J. Hypothalamic regulation of sleep and circadian rhythms. Nature. 2005;437(7063):1257-1263.

17. Luo AH, Aston-Jones G. Circuit projection from suprachiasmatic nucleus to ventral tegmental area: a novel circadian output pathway. Eur J Neurosci. 2009;29(4):748-760.

18. Kalsbeek A, et al. SCN outputs and the hypothalamic balance of life. J Biol Rhythms. 2006;21(6):458-469.

19. Chemelli RM, et al. Narcolepsy in orexin knockout mice: molecular genetics of sleep regulation. Cell. 1999;98(4):437-451.

20. Lin L, et al. The sleep disorder canine narcolepsy is caused by a mutation in the hypocretin (orexin) receptor 2 gene. Cell. 1999;98(3):365-376.

21. Morton GJ, Cummings DE, Baskin DG, Barsh GS, Schwartz MW. Central nervous system control of food intake and body weight. Nature. 2006;
443(7109):289-295

22. Saper CB, Chou TC, Elmquist JK. The need to feed: homeostatic and hedonic control of eating. Neuron. 2002;36(2):199-211.

23. Gao Q, Horvath TL. Neurobiology of feeding and energy expenditure. Annu Rev Neurosci. 2007; 30:367-398.

24. Cone RD. Anatomy and regulation of the central melanocortin system. Nat Neurosci. 2005; 8(5):571-578.

25. Arble DM, Bass J, Laposky AD, Vitaterna MH, Turek FW. Circadian timing of food intake contributes to weight gain. Obesity (Silver Spring). 2009; 17(11):2100-2102.

26. Yoshida K, McCormack S, Espana RA, Crocker A, Scammell TE. Afferents to the orexin neurons of the rat brain. J Comp Neurol. 2006;494(5):845-861.

27. Sakurai T. The neural circuit of orexin (hypocretin): maintaining sleep and wakefulness. Nat Rev Neurosci. 2007;8(3):171-181.

28. Yi CX, et al. A major role for perifornical orexin neurons in the control of glucose metabolism in rats. Diabetes. 2009;58(9):1998-2005.

29. Funato H, et al. Enhanced orexin receptor-2 signaling prevents diet-induced obesity and improves leptin sensitivity. Cell Metab. 2009;9(1):64-76.

30. Wise RA. Brain reward circuitry: insights from unsensed incentives. Neuron. 2002;36(2):229-240.

31. Szczypka MS, Mandel RJ, Donahue BA, Snyder RO, Leff SE, Palmiter RD. Viral gene delivery selectively restores feeding and prevents lethality of dopamine-deficient mice. Neuron. 1999;22(1):167-178.

32. Hampp G, et al. Regulation of monoamine oxidase A by circadian-clock components implies clock influence on mood. Curr Biol. 2008;18(9):678-683.

33. Yamazaki S, et al. Resetting central and peripheral circadian oscillators in transgenic rats. Science. 2000;288(5466):682-685.

34. Yoo SH, et al. PERIOD2:LUCIFERASE real-time reporting of circadian dynamics reveals persistent circadian oscillations in mouse peripheral tissues. Proc Natl Acad Sci U S A. 2004;101(15):5339-5346.

35. Balsalobre A, Damiola F, Schibler U. A serum shock induces circadian gene expression in mammalian tissue culture cells. Cell. 1998;93(6):929-937.

36. Kornmann B, Schaad O, Bujard H, Takahashi JS, Schibler U. System-driven and oscillator-dependent circadian transcription in mice with a conditionally active liver clock. PLoS Biol. 2007;5(2):e34.

37. Damiola F, Le Minh N, Preitner N, Kornmann B, Fleury-Olela F, Schibler U. Restricted feeding uncouples circadian oscillators in peripheral tissues from the central pacemaker in the suprachiasmatic nucleus. Genes Dev. 2000;14(23):2950-2961.

38. Stokkan KA, Yamazaki S, Tei H, Sakaki Y, Menaker $\mathrm{M}$. Entrainment of the circadian clock in the liver by feeding. Science. 2001;291(5503):490-493.

39. Buhr ED, Yoo SH, Takahashi JS. Temperature as a universal resetting cue for mammalian circadian oscillators. Science. 2010;330(6002):379-385.

40. Balsalobre A, et al. Resetting of circadian time in peripheral tissues by glucocorticoid signaling. Science. 2000;289(5488):2344-2347.

41. Rutter J, Reick M, Wu LC, McKnight SL. Regulation of clock and NPAS2 DNA binding by the redox state of NAD cofactors. Science. 2001; 293(5529):510-514.

42. Nemoto S, Fergusson MM, Finkel T. Nutrient avail- ability regulates SIRT1 through a forkhead-dependent pathway. Science. 2004;306(5704):2105-2108.

43. Cohen HY, et al. Calorie restriction promotes mammalian cell survival by inducing the SIRT1 deacetylase. Science. 2004;305(5682):390-392.

44. Asher G, et al. SIRT1 regulates circadian clock gene expression through PER2 deacetylation. Cell. 2008;134(2):317-328.

45. Nakahata Y, et al. The NAD+-dependent deacetylase SIRT1 modulates CLOCK-mediated chromatin remodeling and circadian control. Cell. 2008; 134(2):329-340.

46. Nakahata Y, Sahar S, Astarita G, Kaluzova M, Sassone-Corsi P. Circadian control of the NAD+ salvage pathway by CLOCK-SIRT1. Science. 2009; 324(5927):654-657.

47. Ramsey KM, et al. Circadian clock feedback cycle through NAMPT-mediated NAD+ biosynthesis. Science. 2009;324(5927):651-654.

48. Kahn BB, Alquier T, Carling D, Hardie DG. AMPactivated protein kinase: ancient energy gauge provides clues to modern understanding of metabolism. Cell Metab. 2005;1(1):15-25.

49. Lamia KA, et al. AMPK regulates the circadian clock by cryptochrome phosphorylation and degradation. Science. 2009;326(5951):437-440.

50. Canto C, et al. AMPK regulates energy expenditure by modulating NAD+ metabolism and SIRT1 activity. Nature. 2009;458(7241):1056-1060.

51. Yang X, et al. Nuclear receptor expression links the circadian clock to metabolism. Cell. 2006; 126(4):801-810.

52. Duez H, Staels B. Rev-erb alpha gives a time cue to metabolism. FEBS Lett. 2008;582(1):19-25.

53 . Yin L, et al. Rev-erbalpha, a heme sensor that coordinates metabolic and circadian pathways. Science. 2007;318(5857):1786-1789.

54. Sato TK, et al. A functional genomics strategy reveals Rora as a component of the mammalian circadian clock. Neuron. 2004;43(4):527-537.

55. Lau P, Fitzsimmons RL, Raichur S, Wang SC, Lechtken A, Muscat GE. The orphan nuclear receptor, RORalpha, regulates gene expression that controls lipid metabolism: staggerer (SG/SG) mice are resistant to diet-induced obesity. J Biol Chem. 2008;283(26):18411-18421.

56. Lefebvre P, Chinetti G, Fruchart JC, Staels B. Sorting out the roles of PPAR alpha in energy metabolism and vascular homeostasis. J Clin Invest. 2006;116(3):571-580.

57. Canaple L, et al. Reciprocal regulation of brain and muscle Arnt-like protein 1 and peroxisome proliferator-activated receptor alpha defines a novel positive feedback loop in the rodent liver circadian clock. Mol Endocrinol. 2006;20(8):1715-1727.

58. Semple RK, Chatterjee VK, O'Rahilly S. PPAR gamma and human metabolic disease. J Clin Invest. 2006;116(3):581-589.

59. Wang N, et al. Vascular PPARgamma controls circadian variation in blood pressure and heart rate through Bmal1. Cell Metab. 2008;8(6):482-491.

60. Lopez-Molina L, Conquet F, Dubois-Dauphin M, Schibler U. The DBP gene is expressed according to a circadian rhythm in the suprachiasmatic nucleus and influences circadian behavior. EMBOJ. 1997;16(22):6762-6771.

61. Lin J, Handschin C, Spiegelman BM. Metabolic control through the PGC-1 family of transcription 
coactivators. Cell Metab. 2005;1(6):361-370.

62. Liu C, Li S, Liu T, Borjigin J, Lin JD. Transcriptional coactivator PGC-1alpha integrates the mammalian clock and energy metabolism. Nature. 2007;447(7143):477-481.

63. Picard F, et al. Sirt 1 promotes fat mobilization in white adipocytes by repressing PPAR-gamma. Nature. 2004;429(6993):771-776.

64. Rodgers JT, Lerin C, Haas W, Gygi SP, Spiegelman BM, Puigserver P. Nutrient control of glucose homeostasis through a complex of PGC-1alpha and SIRT1. Nature. 2005;434(7029):113-118.

65. Lam TK, Gutierrez-Juarez R, Pocai A, Rossetti L. Regulation of blood glucose by hypothalamic pyruvate metabolism. Science. 2005;309(5736):943-947.

66. Davis JD, Wirtshafter D, Asin KE, Brief D. Sustained intracerebroventricular infusion of brain fuels reduces body weight and food intake in rats. Science. 1981;212(4490):81-83.

67. Acheson KJ, Ravussin E, Wahren J, Jequier E. Thermic effect of glucose in man. Obligatory and facultative thermogenesis. J Clin Invest. 1984;74(5):1572-1580.

68. Lam TK, Schwartz GJ, Rossetti L. Hypothalamic sensing of fatty acids. Nat Neurosci. 2005;8(5):579-584.

69. Loftus TM, et al. Reduced food intake and body weight in mice treated with fatty acid synthase inhibitors. Science. 2000;288(5475):2379-2381.

70. Pocai A, et al. Restoration of hypothalamic lipid sensing normalizes energy and glucose homeostasis in overfed rats. J Clin Invest. 2006;116(4):1081-1091.

71. Kohsaka A, et al. High-fat diet disrupts behavioral and molecular circadian rhythms in mice. Cell Metab. 2007;6(5):414-421.

72. Minokoshi Y, et al. Leptin stimulates fatty-acid oxidation by activating AMP-activated protein kinase. Nature. 2002;415(6869):339-343.

73. Minokoshi Y, et al. AMP-kinase regulates food intake by responding to hormonal and nutrient signals in the hypothalamus. Nature. 2004; 428(6982):569-574.

74. Claret M, et al. AMPK is essential for energy homeostasis regulation and glucose sensing by POMC and AgRP neurons. J Clin Invest. 2007;117(8):2325-2336.

75. Satoh A, et al. SIRT1 promotes the central adaptive response to diet restriction through activation of the dorsomedial and lateral nuclei of the hypothalamus. J Neurosci. 2010;30(30):10220-10232.

76. Dietrich MO, et al. Agrp neurons mediate Sirt1's action on the melanocortin system and energy balance: roles for Sirt 1 in neuronal firing and synaptic plasticity. J Neurosci. 2010;30(35):11815-11825

77. Cota D, et al. Hypothalamic mTOR signaling regulates food intake. Science. 2006;312(5775):927-930.

78. Cao R, Li A, Cho HY, Lee B, Obrietan K. Mammalian target of rapamycin signaling modulates photic entrainment of the suprachiasmatic circadian clock. J Neurosci. 2010;30(18):6302-6314

79. Ding JM, et al. A neuronal ryanodine receptor mediates light-induced phase delays of the circadian clock. Nature. 1998;394(6691):381-384.

80. Vollmers C, Gill S, DiTacchio L, Pulivarthy SR, Le $\mathrm{HD}$, Panda S. Time of feeding and the intrinsic circadian clock drive rhythms in hepatic gene expression. Proc Natl Acad Sci U S A. 2009;106(50):21453-21458.

81 . Oster $\mathrm{H}$, et al. The circadian rhythm of glucocorticoids is regulated by a gating mechanism residing in the adrenal cortical clock. Cell Metab. 2006; 4(2):163-173.

82. Van Cauter E, Polonsky KS, Scheen AJ. Roles of circadian rhythmicity and sleep in human glucose regulation. Endocr Rev. 1997;18(5):716-738.

83. Sinha MK, et al. Nocturnal rise of leptin in lean, obese, and non-insulin-dependent diabetes mellitus subjects. J Clin Invest. 1996;97(5):1344-1347.

84. Dupuis J, et al. New genetic loci implicated in fasting glucose homeostasis and their impact on type 2 diabetes risk. Nat Genet. 2010;42(2):105-116.

85 . Woon PY, et al. Aryl hydrocarbon receptor nuclear translocator-like (BMAL1) is associated with susceptibility to hypertension and type 2 diabetes. Proc Natl Acad Sci U S A. 2007;104(36):14412-14417.

86. Scott EM, Carter AM, Grant PJ. Association between polymorphisms in the Clock gene, obesity and the metabolic syndrome in man. Int J Obes (Lond). 2008;32(4):658-662.

87. Spiegel K, Tasali E, Leproult R, Van Cauter E. Effects of poor and short sleep on glucose metabolism and obesity risk. Nat Rev Endocrinol. 2009;5(5):253-261

88. Scheer FA, Hilton MF, Mantzoros CS, Shea SA Adverse metabolic and cardiovascular consequences of circadian misalignment. Proc Natl Acad Sci U S A. 2009;106(11):4453-4458

89. Boden G, Chen X, Polansky M. Disruption of circadian insulin secretion is associated with reduced glucose uptake in first-degree relatives of patients with type 2 diabetes. Diabetes. 1999;48(11):2182-2188.

90. Toh KL, et al. An hPer2 phosphorylation site mutation in familial advanced sleep phase syndrome. Science. 2001;291(5506):1040-1043.

91. Xu Y, et al. Functional consequences of a CKIdelta mutation causing familial advanced sleep phase syndrome. Nature. 2005;434(7033):640-644.

92. Jones CR, et al. Familial advanced sleep-phase syndrome: A short-period circadian rhythm variant in humans. Nat Med. 1999;5(9):1062-1065.

93. Bass J, Turek FW. Sleepless in America: a pathway to obesity and the metabolic syndrome? Arch Intern Med. 2005;165(1):15-16

94. Taheri S, Lin L, Austin D, Young T, Mignot E. Short sleep duration is associated with reduced leptin, elevated ghrelin, and increased body mass index. PLoS Med. 2004;1(3):e62.

95. Spiegel K, Leproult R, Van Cauter E. Impact of sleep debt on metabolic and endocrine function. Lancet. 1999;354(9188):1435-1439.

96. Nedeltcheva AV, Kilkus JM, Imperial J, Schoeller DA, Penev PD. Insufficient sleep undermines dietary efforts to reduce adiposity. Ann Intern Med. 2010;153(7):435-441.

97. Thannickal TC, et al. Reduced number of hypocretin neurons in human narcolepsy. Neuron. 2000;27(3):469-474.

98. Peyron C, et al. A mutation in a case of early onset narcolepsy and a generalized absence of hypocretin peptides in human narcoleptic brains. Nat Med. 2000;6(9):991-997.

99. Schuld A, Hebebrand J, Geller F, Pollmacher T Increased body-mass index in patients with narcolepsy. Lancet. 2000;355(9211):1274-1275.

100.Kok SW, et al. Hypocretin deficiency in narcoleptic humans is associated with abdominal obesity. Obes Res. 2003;11(9):1147-1154.

101. Kotagal S, Krahn LE, Slocumb N. A putative link between childhood narcolepsy and obesity. Sleep Med. 2004:5(2):147-150.

102. Howell MJ, Schenck CH, Crow SJ. A review of nighttime eating disorders. Sleep Med Rev. 2009; 13(1):23-34.

103. Colles SL, Dixon JB, O’Brien PE. Night eating syndrome and nocturnal snacking: association with obesity, binge eating and psychological distress. Int J Obes (Lond). 2007;31(11):1722-1730.

104. Birketvedt GS, et al. Behavioral and neuroendocrine characteristics of the night-eating syndrome. JAMA. 1999;282(7):657-663.

105. O'Reardon JP, et al. Circadian eating and sleeping patterns in the night eating syndrome. Obes Res. 2004; 12(11):1789-1796.

106. Goel N, et al. Circadian rhythm profiles in women with night eating syndrome. J Biol Rhythms. 2009 24(1):85-94.

107. Turek FW, et al. Obesity and metabolic syndrome in circadian Clock mutant mice. Science. 2005 308(5724):1043-1045.

108.Salgado-Delgado R, Angeles-Castellanos M, Saderi N,
Buijs RM, Escobar C. Food intake during the normal activity phase prevents obesity and circadian desynchrony in a rat model of night work. Endocrinology. 2010;151(3):1019-1029.

109. Masaki T, et al. Involvement of hypothalamic histamine $\mathrm{H} 1$ receptor in the regulation of feeding rhythm and obesity. Diabetes. 2004;53(9):2250-2260.

110. Oishi K, et al. Disrupted fat absorption attenuates obesity induced by a high-fat diet in Clock mutant mice. FEBS Lett. 2006;580(1):127-130.

111. Oishi $\mathrm{K}$, et al. CLOCK is involved in obesityinduced disordered fibrinolysis in ob/ob mice by regulating PAI-1 gene expression. J Thromb Haemost. 2006;4(8):1774-1780.

112.Dudley CA, et al. Altered patterns of sleep and behavioral adaptability in NPAS2-deficient mice. Science. 2003;301(5631):379-383.

113. Naylor E, et al. The circadian clock mutation alters sleep homeostasis in the mouse. J Neurosci. 2000;20(21):8138-8143.

114.Lamia KA, Storch KF, Weitz CJ. Physiological significance of a peripheral tissue circadian clock. Proc Natl Acad Sci U S A. 2008;105(39):15172-15177.

115. Rudic RD, et al. BMAL1 and CLOCK, two essential components of the circadian clock, are involved in glucose homeostasis. PLoS Biol. 2004;2(11):e377.

116. McDearmon EL, et al. Dissecting the functions of the mammalian clock protein BMAL1 by tissue-specific rescue in mice. Science. 2006;314(5803):1304-1308.

117. Andrews JL, et al. CLOCK and BMAL1 regulate MyoD and are necessary for maintenance of skeletal muscle phenotype and function. Proc Natl Acad Sci U S A. 2010;107(44):19090-19095.

118.Yang $\mathrm{S}$, et al. The role of $\mathrm{mPer} 2$ clock gene in glucocorticoid and feeding rhythms. Endocrinology. 2009; 150(5):2153-2160.

119.la Fleur SE, Kalsbeek A, Wortel J, Fekkes ML, Buijs RM. A daily rhythm in glucose tolerance: a role for the suprachiasmatic nucleus. Diabetes. 2001;50(6):1237-1243.

120. Kalsbeek A, Yi CX, La Fleur SE, Fliers E. The hypothalamic clock and its control of glucose homeostasis. Trends Endocrinol Metab. 2010;21(7):402-410.

121. Boden G, Ruiz J, Urbain JL, Chen X. Evidence for a circadian rhythm of insulin secretion. Am J Physiol. 1996;271(2 pt 1):E246-E252.

122.Feneberg R, Lemmer B. Circadian rhythm of glucose uptake in cultures of skeletal muscle cells and adipocytes in Wistar-Kyoto, Wistar, Goto-Kakiza$\mathrm{ki}$, and spontaneously hypertensive rats. Chronobiol Int. 2004;21(4-5):521-538.

123. Marcheva B, et al. Disruption of the clock components CLOCK and BMAL1 leads to hypoinsulinaemia and diabetes. Nature. 2010;466(7306):627-631.

124.Zhang EE, et al. Cryptochrome mediates circadian regulation of cAMP signaling and hepatic gluconeogenesis. Nat Med. 2010;16(10):1152-1156.

125. Cailotto $\mathrm{C}$, et al. The suprachiasmatic nucleus controls the daily variation of plasma glucose via the autonomic output to the liver: are the clock genes involved? Eur J Neurosci. 2005;22(10):2531-2540.

126. Hussain MM, Pan X. Clock genes, intestinal transport and plasma lipid homeostasis. Trends Endocrinol Metab. 2009;20(4):177-185.

127. Gomez-Abellan P, et al. Circadian expression of adiponectin and its receptors in human adipose tissue. Endocrinology. 2010;151(1):115-122.

128. Licinio J, et al. Synchronicity of frequently sampled, 24-h concentrations of circulating leptin, luteinizing hormone, and estradiol in healthy women. Proc Natl Acad Sci U S A. 1998;95(5):2541-2546.

129.Pan X, Hussain MM. Clock is important for food and circadian regulation of macronutrient absorption in mice. J Lipid Res. 2009;50(9):1800-1813.

130.Pan X, Zhang Y, Wang L, Hussain MM. Diurnal regulation of MTP and plasma triglyceride by CLOCK is mediated by SHP. Cell Metab. 2010;12(2):174-186. 
131. Le Martelot G, et al. REV-ERBalpha participates in circadian SREBP signaling and bile acid homeostasis. PLoS Biol. 2009;7(9):e1000181.

132. Shimba S, et al. Brain and muscle Arnt-like protein-1 (BMAL1), a component of the molecular clock, regulates adipogenesis. Proc Natl Acad Sci U S A. 2005; 102(34):12071-12076.

133. Kalsbeek A, et al. The suprachiasmatic nucleus generates the diurnal changes in plasma leptin levels. Endocrinology. 2001;142(6):2677-2685.

134.Ahima RS, Prabakaran D, Flier JS. Postnatal leptin surge and regulation of circadian rhythm of leptin by feeding. Implications for energy homeostasis and neuroendocrine function. J Clin Invest. 1998; 101(5):1020-1027.

135.Laposky AD, Bradley MA, Williams DL, Bass J,
Turek FW. Sleep-wake regulation is altered in leptin-resistant $(\mathrm{db} / \mathrm{db})$ genetically obese and diabetic mice. Am J Physiol Regul Integr Comp Physiol. 2008;295(6):R2059-R2066.

136.Laposky AD, Shelton J, Bass J, Dugovic C, Perrino $\mathrm{N}$, Turek FW. Altered sleep regulation in leptindeficient mice. Am J Physiol Regul Integr Comp Physiol. 2006;290(4):R894-R903.

137. So AY, Bernal TU, Pillsbury ML, Yamamoto KR, Feldman BJ. Glucocorticoid regulation of the circadian clock modulates glucose homeostasis. Proc Natl Acad Sci U S A. 2009;106(41):17582-17587.

138. Sutton GM, et al. The melanocortin-3 receptor is required for entrainment to meal intake. J Neurosci. 2008;28(48):12946-12955.

139.Saper CB, Fuller PM. Inducible clocks: living in an unpredictable world. Cold Spring Harb Symp Quant Biol. 2007;72:543-550.

140.Stephan FK. The "other" circadian system: food as a Zeitgeber. J Biol Rhythms. 2002;17(4):284-292.

141. Gooley JJ, Schomer A, Saper CB. The dorsomedial hypothalamic nucleus is critical for the expression of food-entrainable circadian rhythms. Nat Neurosci. 2006;9(3):398-407.

142.Fuller PM, Lu J, Saper CB. Differential rescue of light- and food-entrainable circadian rhythms. Science. 2008;320(5879):1074-1077.

143.Landry GJ, Simon MM, Webb IC, Mistlberger RE. Persistence of a behavioral food-anticipatory circadian rhythm following dorsomedial hypothalamic ablation in rats. Am J Physiol Regul Integr Comp Physiol. 2006;290(6):R1527-R1534. 